J Cell Biochem. 2017 November ; 118(11): 3586-3594. doi:10.1002/jcb.26099.

\title{
CRISPR editing technology in biological and biomedical investigation
}

\author{
Martyn K. White, Rafal Kaminski, Won-Bin Young, Pamela C Roehm, and Kamel Khalili ${ }^{\dagger}$ \\ Center for Neurovirology and Comprehensive NeuroAIDS Center, Department of Neuroscience, \\ Lewis Katz School of Medicine at Temple University, 3500 N. Broad Street, Philadelphia, PA \\ 19140, USA
}

\begin{abstract}
The CRISPR or clustered regularly interspaced short palindromic repeats system is currently the most advanced approach to genome editing and is notable for providing an unprecedented degree of specificity, effectiveness and versatility in genetic manipulation. CRISPR evolved as a prokaryotic immune system to confer provide an acquired immunity and resistance to foreign genetic elements such as bacteriophages. It has recently been developed into a tool for the specific targeting of nucleotide sequences within complex eukaryotic genomes for the purpose of genetic manipulation. The power of CRISPR lies in its simplicity and ease of use, its flexibility to be targeted to any given nucleotide sequence by the choice of an easily synthesized guide RNA, and its ready ability to continue to undergo technical improvements. Applications for CRISPR are numerous including creation of novel transgenic cell animals for research, high-throughput screening of gene function, potential clinical gene therapy and nongene-editing approaches such as modulating gene activity and fluorescent tagging. In this prospect article, we will describe the salient features of the CRISPR system with an emphasis on important drawbacks and considerations with respect to eliminating off-target events and obtaining efficient CRISPR delivery. We will discuss recent technical developments to the system and we will illustrate some of the most recent applications with an emphasis on approaches to eliminate human viruses including HIV-1, JCV and HSV-1 and prospects for the future.
\end{abstract}

\section{Keywords}

CRISPR; Cas9; Genome Editing

\section{INTRODUCTION}

Over the last several decades, molecular tools have been developed from the immune defenses of prokaryotic organisms that have allowed the specific manipulation of the genetic material of eukaryote organisms for a variety of purposes, beginning with restriction endonucleases in the 1970s to the continuing present-day advancements of complex strategies. The most notable recent approach is CRISPR editing, which provides unprecedented specificity, effectiveness and versatility in genetic manipulation. CRISPR/Cas

\footnotetext{
${ }^{\dagger}$ Corresponding Author: Tel: 215-707-4500, Fax: 215-707-4888, kamel.kalili@ temple.edu.
} 
or clustered regularly interspaced short palindromic repeats (CRISPR)/CRISPR-associated system is a prokaryotic immune system that confers resistance to foreign genetic elements such as bacteriophages and provides an acquired immunity to them (Mohanraju et al., 2016). CRISPR is one of three main categories of reagents that have recently been developed that are specifically able to target nucleotide sequences within complex eukaryotic genomes. The other two classes are the zinc-finger nucleases (ZFN), which have the FokI endonuclease domain combined with a zinc-finger targeting domain (Jo et al., 2015) and the transcription factor-like effector nucleases (TALEN), which also have FokI endonuclease domain but combined with a targeting domain derived from TAL effector proteins from Xanthomonas spp. (Wright et al., 2014). ZFN, TALEN and CRISPR endonucleases cut through both of the strands of the DNA double helix at their cleavage site to yield a double-strand break (DSB), which is often repaired by nonhomologous end joining (NHEJ). NHEJ is an error prone DNA repair process that may lead to the introduction of small insertion/deletion (InDel) mutations at the targeted location. If two DSBs are introduced, NHEJ may join flanking breaks giving rise to deletion of the region lying in between (White et al., 2015).

Of the three gene editing approaches, CRISPR is the most powerful because of its simplicity and ease of use, its adaptability and flexibility to be targeted to different nucleotide sequences and its continuing technical improvements (White et al., 2015; Liu and Shui, 2016; Ran, 2016; Singh et al., 2016; Komer et al., 2017; Nakade et al., 2017). For ZFN and TALEN, a specific protein is required for each target while for CRISPR, the target specificity is determined by the sequence of a guide RNA (gRNA). For example, CRISPR/ Cas9 is a two component system of the endonuclease enzyme (Cas9) and the gRNA, which is designed with a 20 nucleotide sequence that is involved in the recruitment of the Cas9/ gRNA complex to its target DNA via Watson/Crick base-pairing plus another nucleotide motif known as the protospacer-adjacent motif (PAM). PAM is a trinucleotide sequence that follows immediately after the target sequence and is required for the binding of Cas9 to its target and target cleavage. This occurs 3-4 base pairs upstream to generate a DSB. As mentioned, DSB repair by NHEJ can introduce InDels, which can destroy the open reading frame of target genes. Since specific targeting is provided by a gRNA, which can be chemically synthesized, transcribed in vitro or expressed in cells, CRISPR is a highly specific tool that is easy to use and thus applications have become widespread with over 3,500 published reports in the last two years alone.

The applications for which CRISPR can be used are many and diverse and include the development of potential gene therapies, the creation of novel cell lines and transgenic animals for research, high-throughput screening of genes, and nongene-editing approaches such as modulation of gene expression and fluorescent tagging of chromosomes (Doetschman and Georgieva, 2017). For example, exciting new possibilities have been opened for genetic targeting in mouse embryonic stem cells to generate novel mouse strains (Doetschman and Georgieva, 2017). In this review, we will examine the use of the CRISPR system with a focus on important drawbacks and considerations, especially related to the problems of off-target events and CRISPR delivery, the most recent technical developments to the system, some recent applications and prospects for the future. 


\section{OFF-TARGET CONSIDERATIONS}

Despite the high degree of specificity of the CRISPR system, the induction of mutations at sites other than the intended target is still a major concern, especially in the context of the development of therapeutic clinical applications (Zhang et al., 2015b). The targeting specificity of Cas9 is conferred by the 20 nucleotide guide sequence of the gRNA and PAM but potential off-target cleavage can occur with even a 3 to 5 base mismatch (Zhang et al., 2015b). Identifying off-target cleavage is a formidable problem given the size and complexity of the eukaryotic genome. One widely used strategy is to employ online software programs to predict in silico potential sites (Yee, 2016). This is usually based on homology searching of genome databases with the target sequence and ranking based on the number and position of mismatches, which has been used successfully to identify off-target cleavages in many studies. For example in a CRISPR-based strategy against Polyomavirus JC (JCV), we used this approach to identify host genomic sites that were most closely related to our viral target sequence. PCR products were amplified from the top three most homologous genes and examined for InDel mutations using the SURVEYOR assay, which revealed no cleavage of the off-target genes (Wollebo et al., 2015a). However, the approach of examining homologous genes is biased and is limited by factors that cannot be predicted, e.g., epigenetic modifications (Yee, 2016). Hence several unbiased strategies have been developed to determine off-target effects and these include: marking of DSBs with an integration-defective lentiviral vector (IDLV) and mapping by nonrestrictive linear amplification-mediated PCR (nrLAM-PCR); genome-wide unbiased identification of DSBs enabled by sequencing (GUIDE-seq); high-throughput, genome-wide, translocation sequencing (HTGTS); direct in situ breaks-labeling on streptavidin and next-generation sequencing (BLESS); and in vitro Cas9-digested whole genome sequencing (digenome-seq). The complex technical details of these techniques and their applications have recently been reviewed (Yee, 2016; Zischewski et al., 2017). The recent development of these multiple, sensitive and unbiased methods of off-target effects of CRISPR represents major progress in overcoming this issue.

A significant factor in overcoming off-target effects is the development of better optimized nucleases (Tsai and Joung, 2016; Tycko et al., 2016). In general, there are two ways to reduce off-target effects: increase the specificity of the nuclease cleavage or limit the duration of nuclease expression. One way to increase the cleavage specificity is to make a truncated gRNA (tru-gRNA) by removing 2-3 nucleotides at the PAM-distal end while adding two $\mathrm{G}$ residues at the $5^{\prime}$ end has also been reported to increase specificity (Tsai and Joung, 2016). Another strategy has been to use paired Cas9 nickases (Cas9n) where one of the two endonuclease domains has been catalytically inactivated by mutation. Alternatively, a catalytically inactive Cas9 (dCas9) can be fused to the dimerization-dependent FokI nuclease to form a dimeric gRNA-dependent FokI-dCas9 nucleases, which are currently undergoing evaluation (Tsai and Joung, 2016). Variants of Cas9 have been engineered that have improved specificities, e.g., high-fidelity Cas9 variant 1 (SpCas9-HF1, Kleinstiver et al., 2016) and enhanced Cas9 version 1.1 (eSpCas9 1.1, Slaymaker et al., 2016).

Off-target effects of CRISPR can also be reduced by limiting the duration of Cas9 activity. For example, Cas9-gRNA ribonucleoprotein (RNP) complexes delivered by electroporation 
have a shorter half-life than Cas9 gRNAs delivered by plasmid transfection or lentiviral transduction. Cas9 dosage also affects the kinetics and degree of specificity of cleavage highlighting the importance of titrating delivery to optimize the specificity needed for an application. Specificity can also be increased by using an inducible form of Cas9. For example, Davis et al. (2015) developed an inducible Cas9 activated by a 4hydroxytamoxifen-responsive intein, which modified target sites with $\sim 25$-fold higher specificity than wild-type Cas9 although on-target efficiency was also somewhat reduced. Recently, four CRISPR-Cas9 inhibitor proteins were discovered in Listeria monocytogenes bacteriophage that prevent Cas9 binding and gene editing (Rauch et al., 2017). These represent natural "anti-CRISPRs" tools that can be used to regulate Cas9 genome engineering, i.e., a post-translational Cas9 "off-switch", which could be used to specifically limit the amount of time that Cas9 is active and thus reduce off-target effects. In a more specific strategy, Kaminski et al. (2016c) developed a new approach that turns on Cas expression by the target gene. In this case, Cas9 was under the control of a promoter that is activated by the HIV-1 transactivator protein Tat allowing targeting of HIV-1 only during the course of viral infection when Tat is expressed (Kaminski et al., 2016c).

\section{METHODS OF DELIVERY}

Perhaps the most important issue in CRISPR applications is that of delivery (Liu and Shui, 2016; Wang et al., 2016b). A variety of methods may be used to deliver CRISPR including physical methods and viral and non-viral vectors. Physical methods include transfection, electroporation and microinjection, which are mainly suited to research studies in cell culture but can be used for ex vivo cell manipulation for adoptive transfer. Liang et al. (2015) described delivery of Cas9 protein/gRNA ribonucleoprotein complexes into a variety of mammalian cells using liposome-mediated transfection or electroporation. They reported nuclease-induced InDel rates of up to $87 \%$ in induced pluripotent stem cells (iPSC). In addition, off-target cleavage was reduced when compared to plasmid DNA transfection (Liang et al., 2015). This is presumably because Cas9-RNP complexes delivered by electroporation have a shorter half-life than Cas 9 gRNAs delivered by plasmid transfection or lentiviral transduction. Furthermore, Cas9-RNPs are active shortly after delivery because there is no lag while protein is synthesized. It has also been reported that efficient delivery of Cas9-RNP can be achieved using lipid nanoparticles (Wang et al., 2016c).

Viral vectors for delivery in clinical trials include lentivirus, adenovirus and adenoassociated virus (AAV) vectors. Lentiviral vectors are derived from HIV, provide efficient and stable delivery and can be used to infect both dividing and non-dividing cells, such as the brain cells. Viral genes that are not required for packaging, e.g., nef, vif, vpr, etc., are deleted and the packaging genes are expressed from separate plasmids to reduce the chance of reconstitution of wild-type virus (Liu and Shui, 2016). However because of integration, lentiviral vectors are unsuitable for therapeutic applications but this risk can be reduced by using IDLV (White et al. 2015). Adenoviruses are a family of linear double-stranded DNA viruses about $36 \mathrm{Kbp}$ in length containing four early and five late transcription units (Liu and Shui, 2016). Most vectors are based on adenovirus type 5 (Ad5). Recombinant virus is made by deleting the early gene E1 or E1 plus E3 and grown in a packaging cell line that expresses E1 to produce infectious recombinant virus. Adenovirus can infect both dividing 
and non-dividing cells and does not integrate into host genome. However, both lentivirus and adenovirus vectors have several important drawbacks especially safety concerns related to their immunogenicity (Kotterman et al, 2015). On the other hand, AAV-based vectors are much less immunogenic. AAV is a single-stranded DNA virus of $<4.7 \mathrm{~Kb}$ and like adenovirus requires E1 to package infectious virions, which can transduce both dividing and non-dividing cells. AAV can persist episomally in infected cells but rarely integrates. AAV is a favored vector to use to deliver Cas9 due to its efficiency and low immunogenicity (Senis et al., 2014; Komor et al., 2016). However, the large size of the Cas9 endonuclease is an obstacle to its efficient delivery with the gene for Streptococcus pyogenes Cas9 being about 4.2 Kbp while the maximum cargo size for AAV is about $4.5 \mathrm{Kbp}$ (Ran et al., 2015). Ran et al. (2015) characterized Cas9 orthologues and showed that Cas9 from Streptococcus aureus (SaCas9) has a similar editing efficiency to SpCas9 but is over $1 \mathrm{Kbp}$ shorter and has the potential to execute specific and efficient gene editing. Another Cas orthologue has recently been isolated from Campylobacter jejuni that is even smaller with a size of $2.95 \mathrm{Kbp}$ (CjCas9, Kim et al., 2017). It was shown that the CjCas9 gene could be packaged in an AAV vector together with gRNA and a marker gene to generate a high titer virus that was able to

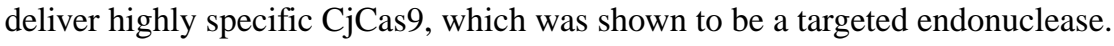

\section{RECENT TECHNICAL DEVELOPMENTS}

The CRISPR system has continued to evolve since its development as a genetic engineering tool about five years ago. Although it is widely used, SpCas9 has disadvantages to which we have already referred, i.e., a high molecular weight and off-targeting events. Here we will describe some recent improvements and alternatives to Cas9 including SpCas9 variants, homologs and other proteins (Ran, 2016; Nakade et al., 2017). To reduce off-target events, variants of SpCas9 have been engineered that have improved specificity, SpCas9-HF1 and eSpCas9 1.1, as described above. In order to increase its versatility, SpCas9 variants have also been engineered that have alternative PAM specificities (Kleinstiver et al., 2015). Other SpCas9 variants mentioned above are Cas9 nickases (Cas9n) and catalytically inactive Cas9 (dCas9). Also of note is the combination of these with cytosine deaminase to produce "base editors" that do not induce DSBs (Nishida et al., 2016; Komor et al., 2016).

While Cas9 exclusively cleaves DNA substrates because of the requirement for PAM for binding and cleavage of the target site, Cas9 is able to bind to single-stranded RNA (ssRNA) targets matching the gRNA sequence when PAM is present in trans on a separate DNA strand known as a PAM-presenting oligonucleotide (PAMmer, O'Connell et al., 2014). PAMmers promote site-specific cleavage of ssRNA targets by Cas9, allowing specific PAMmers to direct binding and cutting of RNA targets while preventing cleavage of corresponding DNA sequences (O'Connell et al., 2014).

In addition to $\mathrm{SpCas} 9$ variants, Cas9 homologs have been isolated from other species such as Streptococcus aureus (SaCas9) and Campylobacter jejuni (CjCas9) as described above. Karvelis et al. (2015) described a novel Cas9 protein from Brevibacillus laterosporus that has a PAM with a broad targeting range and Hirano et al. (2016) described the structure of Cas9 from Francisella novicida FnCas9 and used this structural information to create a variant that can recognize a relaxed $5^{\prime}$-YG-3' PAM. Zetsche et al (2015) reported the 
characterization of a class 2 CRISPR effector, Cpf1 from Acidaminococcus sp. BV3L6 and Lachnospiraceae ND2006, and showed that it has features distinct from Cas9 in that it lacks tracrRNA, uses a T-rich PAM and cleaves DNA via a staggered DNA DSB. Shmakov et al. (2015) described three distinct Class 2 CRISPR-Cas systems: C2c1, C2c2 and C2c3. Interestingly, C2c2 from Leptotrichia shahii is guided by a single CRISPR RNA and can be programmed to cleave single-stranded RNA targets carrying complementary protospacers. Thus C2c2 is a new RNA-targeting tool that could be useful for gene knockdown applications (Abudayyeh et al., 2016). Burstein et al. (2017) adopted a metagenomics approach involving the sequencing of DNA directly extracted from natural microbial communities, providing access to genetic material from a large number of uncultivated organisms and identified a number of novel CRISPR systems, including ones from archaea. Two previously unknown systems were reported, CRISPR-CasX and CRISPR-CasY, which are among the most compact yet discovered. Thus, investigation of environmental microbial communities allows access to an unprecedented diversity of genomes to expand the repertoire of microbe-based biotechnologies. The armamentarium of CRISPR and CRISPRlike systems has become significantly larger recently and will surely continue to grow.

\section{RECENT APPLICATIONS OF CRISPR}

The remarkable versatility, simplicity, specificity and ease of use of CRISPR have led to its use in many thousands of applications with over 4500 published reports mentioning CRISPR in the last three years (Ran, 2015; Shalem et al., 2015; Gaj et al., 2016; Ran, 2016; Singh et al., 2016; Wang et al., 2016d; Wright et al., 2016; Komor et al., 2017). CRISPR has been applied to many disciplines including the genome engineering of animals (Lotti et al., 2016) and plants (Seth and Harish, 2016). In the last several years, new technologies have been developed to increase the ease of applying gene editing technologies to transfer genetic material to livestock animals. These include microinjection, which has proved effective in mice but less so in livestock animals, nuclear transfer, sperm-mediated gene transfer and liposome-mediated DNA transfer (Lotti et al., 2016). These have allowed generating transgenic animals to become more accessible and affordable and enabled production of transgenic animals to investigate their biology as well as increasing the ability to produce more efficient animals that are more resistant to disease. In plants, CRISPR has become a promising tool for the manipulation of agricultural crops, including rice, potato, maize, wheat, barley, soybean, sorghum, cabbage, and tomato (Seth and Harish, 2016) with the aim of improving crop yield and developing crop varieties that are resistant to disease.

The CRISPR approach is also useful for the creation of genetically modified animal models for the study of human diseases (Low et al., 2016), especially by the modification of the mouse genome since mice have a short generation time and are comparatively easy and inexpensive to produce and maintain. Thus mice provide an ideal mammal in which to examine genome functions and complexities. CRISPR can be used to precisely tailor the mouse genome and create models of human diseases that may be progressively refined to better reflect human disease and develop therapeutics (Low et al., 2016). For example, we can improve our knowledge of gene function by the analysis by the knockout of a gene in the mouse (Tschaharganeh et al., 2016) and develop novel therapeutic approaches to important human diseases with a genetic basis including cancer (White and Khalili, 2016). 
Cancer is characterized by the occurrence of multiple mutations and many years of research have allowed the understanding of the different functional classes into which the mutated genes can be grouped. These include the oncogenes, which enhance cellular proliferation, the tumor suppressors, which regulate cell growth potential and metabolic enzymes conferring resistance to chemotherapy. The ability to correct such mutations by genetic alteration is an important objective of cancer therapy and hence CRISPR is a promising tool (White and Khalili, 2016).

The discovery of CRISPR has also made it possible to develop potent new weapons against infectious diseases (Doerflinger et al., 2017). Novel therapeutic options are urgently needed for multidrug-resistant bacteria such as Mycobacterium tuberculosis and for by acute infections such as malaria and dengue fever as well for chronic viral infections such as human immunodeficiency virus (HIV-1) and hepatitis B virus (HBV) as described below. CRISPR/Cas9 libraries can be used to perform whole genome phenotypic to aid in identifying critical host factors that regulate the pathogenesis of infectious agents.

Thus there are many applications for CRISPR that hold much promise for the clinic. In October of 2016, the first clinical trial of CRISPR technology was begun when PD-1 knockout engineered $\mathrm{T}$ cells were injected into human subjects as a therapy for metastatic non-small cell lung cancer (Cyranoski et al., 2016). We have been interested in our research in developing CRISPR/Cas9 approaches to human pathogenic viruses (White et al., 2015, 2016; Khalili et al., 2017), in particular HIV-1, which causes AIDS, and Polyomavirus JC (JCV), which causes progressive multifocal leukoencephalopathy (PML; Wollebo et al., 2015b). We will discuss these studies in the following section as well as CRISPR strategies to other human virus. These illustrate the application of CRISPR technology to human disorders, the problems that may be encountered and possible strategies to overcome them.

\section{Application of CRISPR editing strategy to human viruses}

Antiviral agents that are specifically capable of targeting nucleotide sequences within the genome of a human virus are an attractive prospect for addition to the antiviral arsenal. This is particularly true for viruses that are able to enter a state of latency or persistence where viral gene expression is low or absent and viral proteins may not be present to serve as targets for drugs or vaccination. CRISPR/Cas9 is able to target the genomes of doublestranded DNA viruses and retroviruses, which have a DNA intermediate in their life cycle (White et al., 2015). Now that CRISPR effector endonucleases are available to target RNA, e.g., FnCas9 directed by an engineered RNA-targeting gRNA, inhibition of human RNA viruses such as hepatitis $\mathrm{C}$ virus within eukaryotic cells should be possible (Price et al., 2015). Our research has focused on several pathogenic human viruses, including JCV, which is a circular double-stranded DNA virus (Wollebo et al., 2015b), HIV-1, which is a retrovirus (Hu et al., 2014; Kaminski et al., 2016a,b,c; Khalili et al., 2015, 2017; White and Khalili, 2016; White et al., 2015, 2016; Yin et al., 2016, 2017; Zhang et al., 2015a) and HSV-1 a herpesvirus comprised of a double-stranded DNA of greater than $100 \mathrm{Kbp}$ in size (McGeoch et al., 2006).

JCV causes PML, a lethal CNS demyelinating disease, which ensues when the virus actively replicates in the oligodendrocytes and astrocytes of the central nervous system (CNS) 
producing cytolysis, demyelinated lesions and devastating neurological consequences (Wollebo et al., 2015b). While PML is rare, infection by JCV in the human population is common and virus persists in an asymptomatic or latent state. Thus an approach such as CRISPR/Cas that targets the viral genome is attractive. JCV has a small $(\sim 5 \mathrm{Kbp})$ genome containing an early coding, late coding and noncoding control regions. We have employed CRISPR/Cas9 to introduce mutations in the JCV gene for the essential viral early protein Tantigen (Wollebo et al., 2015a), which interfered with the function of T-antigen and suppressed viral replication with no detectable off-target effects. In a subsequent report, another laboratory used CRISPR/Cas9 to target the control and late regions of JCV and also found significant inhibition of virus replication (Chou et al., 2016). Thus JCV-specific CRISPR/Cas9 gene editing is a promising tool for the elimination of JCV and a potential cure for PML.

Herpes simplex virus-1 (HSV-1) is a human neurotropic virus, which commonly infects many people and persists in a latent stage in cranial nerve ganglia. However in rare cases, HSV-1 can replicate in the central nervous system causing encephalitis. We and several other laboratories applied CRISPR/Cas9 to HSV-1 (Roehm et al., 2016; van Diemen et al., 2016; $\mathrm{Li}$ et al., 2017). We found that CRISPR/Cas9 introduced InDels into the HSV-1 ICP0 gene reducing viral infectivity in human cell cultures and protecting them against HSV-1 infection. CRISPR targeting ICP0 plus the ICP4 or ICP27 completely abrogated HSV-1 infection indicating that CRISPR/Cas9 has the potential to be developed as a novel and specific therapeutic and prophylactic tool for HSV-1 disease (Roehm et al., 2016).

HIV-1/AIDS remains a significant public health problem even though the advent of combination antiretroviral therapy (cART) in the 1990s has meant that HIV is now a chronic condition and progression to AIDS is rare in many regions. However, infected reservoirs, e.g., CD4+T cells, allow virus to persist as an integrated DNA provirus with the potential for reemergence. Different approaches have been employed to eradicate HIV from these reservoirs including the so-called "shock and kill" approach, where latent virus is triggered by cellular reactivation so as to purge virus-producing cells. However, an approach using CRISPR gene-editing for inactivating integrated proviral DNA in the genome of latently infected cells has the potential to eliminate latent viral reservoirs allowing a sterile cure of AIDS (Khalili et al., 2015, 2017; White et al., 2016). CRISPR/Cas9 can be used either to disrupt the integrated HIV-1 provirus or the cellular genes that are required for productive HIV-1 infection. For example, CRISPR/Cas9 facilitates the excision of HIV proviral DNA segments in several different cell types including CD4+ T-cells and by targeting the flanking HIV-1 LTR sequences, complete excision of proviral DNA can be effected (Ebina et al., 2013; Hu et al., 2014; Kaminski et al., 2016b; Yin et al., 2016). This approach has been demonstrated to be effective in cleaving HIV proviral DNA in latently-infected cells in HIVinfected humanized Bone marrow-Liver-Thymus (hu-BLT) mice (Yin et al, 2017). The cleaved HIV proviral DNA appears to be the dominant species over the unedited full length proviral DNA in some tissues, i.e. heart or colon, which is promising for a potential eradication of HIV reservoirs. Prophylactically protection of uninfected cells is also possible by CRISPR/Cas9 expression (Hu et al., 2014; Liao et al., 2015; Kaminski et al., 2016b). However, since extended expression of CRISPR/Cas9 in the cells is necessary with this tactic, off-target effects may become a problem as explained above. A related approach has 
been to use a catalytically-deficient Cas9-synergistic activation mediator (dCas9-SAM) as a means to activate the HIV-1 provirus similar to the "shock and kill" approach only with a novel and more specific viral latency-reversing tool (Zhang et al., 2015a; Bialek et al., 2016).

The strategy of using CRISPR as a weapon against HIV-1 is an attractive approach to eliminate provirus from latently infected cells because the integration of HIV proviral DNA is an important step for HIV replication. Thus, by cleaving the essential HIV-1 genes from the host genome using multiplex gRNAs to target multiple sites of HIV provirus, the function of the target viral gene can be disrupted so as to prohibit HIV replication. However, if only single gRNA is used for a single target site, it is possible to generate InDels where the mutated gene can be changed in such a way as to allow the virus to replicate but yet no longer be susceptible to cleavage by Cas 9 because its mutated sequence is now different to the Cas9/gRNA target sequence, i.e., an "escape mutant". (Wang et al., 2016a, 2016e; Khalili et al., 2017). Thus when designing any gene editing strategy against HIV, it is essential to consider the possibility of escape mutants arising (White et al., 2016). In principle, multiplex gRNAs can be employed so that the chance of generating escape mutants can be greatly reduced, since the probability of viable mutations occurring at multiple sites is much less than for a single site. Also, if the multiplex gRNAs are designed in such a way as to produce DNA breaks resulting in a large section of DNA being deleted, this will permanently prevent escape mutations from occurring (Hu et al., 2014; Kaminski et al., 2016a,b; Yin et al., 2016, 2017). Some Cas9 variants, e.g., Cpf1, cleave at a site that is outside the target sequence so that InDel mutations introduced at the cleavage site by NHEJ will not prevent further cleavage (Zetsche et al., 2015). Another possibility is to combine CRISPR/Cas with other antiviral modalities, e.g., RNA interference (RNAi) or a small molecule antiviral, to apply a multipronged assault. Antiviral-induced reduction of the replication rate of the virus and the viral titer may provide a way to render virus more susceptible to cleavage by CRISPR (White et al., 2016). Finally, another approach that has been applied to HIV-1, and that should not be affected by CRISPR-generated escape mutants, is to disrupt cellular molecules that are necessary for HIV-1 infection, e.g., the coreceptors, CCR5 and CXCR4 (Ye et al., 2014; Hou et al., 2015). Cells become refractory to HIV infection in a way such that CRISPR is not able to generate escape mutants. The application of CRISPR to HIV is an illustrative on-going example of how CRISPR can be used in biomedical investigation, the problems that can arise and how they may be addressed.

CRISPR has also been used as an approach against other human viruses (White et al., 2015). Human papillomaviruses (HPVs) are a proven etiological cause of human cancers and the major cause of cervical carcinoma. The E6 and E7 genes of carcinogenic HPVs affect their oncogenic properties and are thus prime targets for therapeutic intervention in HPVassociated malignant disease. The molecular mechanisms of these two oncogenes have been well defined: E6 binds and degrades p53 and E7 binds pRb, p107 and p130 resulting in phosphorylation and release of the E2F transcription factors. A number of cervical carcinoma cell lines which contain integrated HPV have been treated with Cas9 and E6- or E7-specific gRNAs and show diminished cell proliferation in vitro and dramatic inhibition of tumorigenesis in nude mice. These studies have been reviewed recently and show the 
potential for CRISPR to be developed for clinical therapy of HPV-associated tumors (Kennedy and Cullen, 2017).

CRISPR has also been applied to other DNA viruses of the herpesvirus family as well as HSV-1. Epstein-Barr virus (EBV) causes Burkitt's lymphoma and nasopharyngeal carcinoma and is able to enter a latent state where the circular episomal EBV genome expresses a small number of proteins that are thought to be involved in cellular transformation. Investigations using the CRISPR/Cas9 system have shown potential to reduce cellular proliferation and cause a decline in viral load (White et al., 2015; Yuen et al., 2017).

HBV is a small, circular, partially double-stranded DNA virus that can cause acute and chronic liver infections, which may lead to liver failure, cirrhosis and hepatocellular carcinoma. Chronic infection involves HBV persistence as an episome known as the covalently closed circular DNA (cccDNA), which encodes only a few proteins including HBV Protein X (HBx) and HBV surface antigen (HBsAg) that are important in transformation. Disabling the cccDNA is thus the key to curing HBV infections and CRISPR)/Cas9 has been reported to be efficacious in cultured cells and in mice showing the potential of CRISPR/Cas9 to treat HBV-associated diseases (Moyo et al., 2017). Hepatitis C virus (HCV) is a small, positive-sense, single-stranded RNA flavivirus and can cause hepatocellular carcinoma and lymphomas. Recent advances in drug development have allowed treatment of $\mathrm{HCV}$ infection but nevertheless, targeting HCV RNA from HCV with FnCas9 from Francisella novicida may have therapeutic utility (Moyo et al., 2017).

\section{Other uses of CRISPR/Cas9}

The CRISPR/Cas9 system has many uses that depend on its endonuclease activity. In addition, catalytically inactive Cas9 (dCas9) can be fused to other proteins and used together with gRNA to exert sequence-specific targeted effects such as gene activation or gene silencing. For example, dCas9-SAM can be used as a means to activate HIV-1 provirus as discussed above (Zhang et al., 2015a; Bialek et al., 2016). dCas9 derivatives can also be used to execute target-specific epigenetic changes such as DNA methylation and histone acetylation (Cano-Rodriguez and Rots, 2016). For example, dCas9 has been fused to TET1 DNA demethylase catalytic domain and used to target the BRCA1 promoter where it caused active DNA demethylation and upregulation of BRCA1 (Choudhury et al., 2016). dCas9 derivatives have also been employed to tag chromosome targets so as to reveal nuclear organization and to tag specific mRNAs and track them in cells (Doetschman and Georgieva, 2017). For example, a dCas9-EGFP (enhanced green fluorescent protein) fusion protein was introduced into cells together with tiled gRNAs along a specific chromosomal site to study telomere dynamics (Chen et al., 2013).

\section{CONCLUSIONS AND FUTURE DIRECTIONS}

The CRISPR system is a powerful tool for simple, facile and specific genetic editing of eukaryotic genomes. It has found many potential applications to human diseases including genetic disorders, cancer and viruses. In the last few years, much progress has been made in minimizing off-target effects and addressing problems with delivery. Many of the 
applications of CRISPR have been discussed and will no doubt continue their development toward the clinic. Diseases with a genetic basis such as cancer are amenable to the development of CRISPR-based therapies. As previously noted, the first clinical trial of CRISPR began last fall when CRISPR-engineered PD-1 knockout $T$ cells were used to treat metastatic non-small cell lung cancer (Cyranoski et al., 2016) in China and the first US trials are slated to begin this year. A similar study is planned at the University of Pennsylvania and has been approved by the NIH and is awaiting approval from the FDA. In this trial, T-cells from 18 patients will be edited ex vivo to treat cancers including melanoma, sarcoma and myeloma. CRISPR is also a promising tool for the development of novel therapies to treat inherited disorders such as sickle cell disease. For example, $\beta$-hemoglobinopathies, such as sickle cell anemia and $\beta$-thalassemia, which affect millions of people around the world, are caused by mutations in the gene encoding $\beta$-globin. Dever et al. (2016) have reported that a CRISPR/Cas9 gene-editing system that combined Cas9 ribonucleoprotein and AAV delivery of homologous donor DNA achieved homologous recombination at the human $\beta$-globin gene in hematopoietic stem cells. Enrichment allowed the purification of hematopoietic stem cells with $>90 \%$ targeted integration and correction of the Glu6Val sickle cell mutation and appropriate $\beta$-globin mRNA expression after differentiation into erythrocytes (Dever et al., 2016). These preclinical studies illustrate the feasibility of CRISPR-based methods for $e x$ vivo $\beta$-globin correction in patient-derived stem cells followed by autologous transplantation as a therapy for $\beta$-hemoglobinopathies. Another potential use for CRISPR is to treat retinitis pigmentosa. Retinitis pigmentosa results from loss of cone photoreceptors leading to blindness and is thought to occur secondary to the degeneration of rod photoreceptors. Yu et al (2017) reported mediated CRISPR/Cas9 delivery to postmitotic photoreceptors by AAV to target the Nrl gene, which encodes a neural retina-specific leucine zipper protein and a rod fate determinant during photoreceptor development. Disrupting Nrl enabled rods to gain some of the features of cones and improved their survival hence preventing secondary cone degeneration. Three different mouse models of retinal degeneration exhibited considerable improvement in rod survival and cone function in response to treatment, suggesting that CRISPR-mediated disruption of NRL in rods may be a promising potential therapeutic option for retinitis pigmentosa patients (Yu et al., 2017). Finally, CRISPR may be a potential treatment for Huntington disease. Huntington disease is a dominant neurodegenerative disorder caused by a CAG repeat expansion in HTT. Xu et al. (2017) corrected Huntington patient induced pluripotent stem cells (hiPSCs) with a CRISPR-based approach and showed that they can be differentiated into excitable, synaptically active forebrain neurons that are rescued from disease-associated phenotypic abnormalities such as impaired neural rosette formation, increased susceptibility to growth factor withdrawal, and deficits in mitochondrial respiration. In summary, the CRISPR system continues to undergo technical improvements and the prospects for transition of many applications to the clinic in the near future look promising.

\section{Acknowledgments}

We thank the past and present members of the Department of Neuroscience for their continued support and insightful discussions. We wish to thank Temple medical students, Kamil Amer and Taha Mur, for their contributions to the HSV-1 project. We also acknowledge the intellectual contributions of the Katz School of Medicine at Temple University Comprehensive NeuroAids Center (Basic Science Cores I and II). We are grateful to Cynthia Papaleo for editorial assistance. 


\section{References}

Abudayyeh OO, Gootenberg JS, Konermann S, Joung J, Slaymaker IM, Cox DB, Shmakov S, Makarova KS, Semenova E, Minakhin L, Severinov K, Regev A, Lander ES, Koonin EV, Zhang F. C2c2 is a single-component programmable RNA-guided RNA-targeting CRISPR effector. Science. 2016; 353:aaf5573. [PubMed: 27256883]

Bialek JK, Dunay GA, Voges M, Schäfer C, Spohn M, Stucka R, Hauber J, Lange UC. Targeted HIV-1 latency reversal using CRISPR/Cas9-derived transcriptional activator systems. PLoS One. 2016; 11:e0158294. [PubMed: 27341108]

Burstein D, Harrington LB, Strutt SC, Probst AJ, Anantharaman K, Thomas BC, Doudna JA, Banfield JF. New CRISPR-Cas systems from uncultivated microbes. Nature. 2017; 542:237-241. [PubMed: 28005056]

Cano-Rodriguez D, Rots MG. Epigenetic editing: on the verge of reprogramming gene expression at will. Curr Genet Med Rep. 2016; 4:170-179. [PubMed: 27933223]

Chen B, Gilbert LA, Cimini BA, Schnitzbauer J, Zhang W, Li GW, Park J, Blackburn EH, Weissman JS, Qi LS, Huang B. Dynamic imaging of genomic loci in living human cells by an optimized CRISPR/Cas system. Cell. 2013; 155:1479-1491. [PubMed: 24360272]

Chou YY, Krupp A, Kaynor C, Gaudin R, Ma M, Cahir-McFarland E, Kirchhausen T. Inhibition of JCPyV infection mediated by targeted viral genome editing using CRISPR/Cas9. Sci Rep. 2016; 6:36921. [PubMed: 27841295]

Choudhury SR, Cui Y, Lubecka K, Stefanska B, Irudayaraj J. CRISPR-dCas9 mediated TET1 targeting for selective DNA demethylation at BRCA1 promoter. Oncotarget. 2016; 7:46545-46556. [PubMed: 27356740]

Cyranoski D. CRISPR gene-editing tested in a person for the first time. Nature. 2016; 539:479. [PubMed: 27882996]

Davis KM, Pattanayak V, Thompson DB, Zuris JA, Liu DR. Small molecule-triggered Cas9 protein with improved genome-editing specificity. Nat Chem Biol. 2015; 11:316-318. [PubMed: 25848930]

Dever DP, Bak RO, Reinisch A, Camarena J, Washington G, Nicolas CE, Pavel-Dinu M, Saxena N, Wilkens AB, Mantri S, Uchida N, Hendel A, Narla A, Majeti R, Weinberg KI, Porteus MH. CRISPR/Cas9 $\beta$-globin gene targeting in human haematopoietic stem cells. Nature. 2017; 539:384-389.

Doerflinger M, Forsyth W, Ebert G, Pellegrini M, Herold MJ. CRISPR/Cas9-The ultimate weapon to battle infectious diseases? Cell Microbiol. 2017 (In Press).

Doetschman T, Georgieva T. Gene editing with CRISPR/Cas9 RNA-directed nuclease. Circ Res. 2017; 120:876-894. [PubMed: 28254804]

Ebina H, Misawa N, Kanemura Y, Koyanagi Y. Harnessing the CRISPR/Cas9 system to disrupt latent HIV-1 provirus. Sci Rep. 2013; 3:2510. [PubMed: 23974631]

Gaj T, Sirk SJ, Shui SL, Liu J. Genome-editing technologies: principles and applications. Cold Spring Harb Perspect Biol. 2016; 8 pii:a023754.

Hirano H, Gootenberg JS, Horii T, Abudayyeh OO, Kimura M, Hsu PD, Nakane T, Ishitani R, Hatada I, Zhang F, Nishimasu H, Nureki O. Structure and Engineering of Francisella novicida Cas9. Cell. 2016; 164:950-961. [PubMed: 26875867]

Hou P, Chen S, Wang S, Yu X, Chen Y, Jiang M, Zhuang K, Ho W, Hou W, Huang J, Guo D. Genome editing of CXCR4 by CRISPR/cas9 confers cells resistant to HIV-1 infection. Sci Rep. 2015; 5:15577. [PubMed: 26481100]

Hu W, Kaminski R, Yang F, Zhang Y, Cosentino L, Li F, Luo B, Alvarez-Carbonell D, Garcia-Mesa Y, Karn J, Mo X, Khalili K. RNA-directed gene editing specifically eradicates latent and prevents new HIV-1 infection. Proc Natl Acad Sci USA. 2014; 111:11461-11466. [PubMed: 25049410]

Jinek M, Chylinski K, Fonfara I, Hauer M, Doudna JA, Charpentier E. A programmable dual-RNAguided DNA endonuclease in adaptive bacterial immunity. Science. 2012; 337:816-821. [PubMed: 22745249]

Jo YI, Kim H, Ramakrishna S. Recent developments and clinical studies utilizing engineered zinc finger nuclease technology. Cell Mol Life Sci. 2015; 72:3819-30. [PubMed: 26089249] 
Kaminski R, Bella R, Yin C, Otte J, Ferrante P, Gendelman HE, Li H, Booze R, Gordon J, Hu W, Khalili K. Excision of HIV-1 DNA by gene editing: a proof-of-concept in vivo study. Gene Ther. 2016a; 23:690-695. [PubMed: 27194423]

Kaminski R, Chen Y, Fischer T, Tedaldi E, Napoli A, Zhang Y, Karn J, Hu W, Khalili K. Elimination of HIV-1 genomes from human t-lymphoid cells by CRISPR/Cas9 gene editing. Sci Rep. 2016b; 6:22555. [PubMed: 26939770]

Kaminski R, Chen Y, Salkind J, Bella R, Young WB, Ferrante P, Karn J, Malcolm T, Hu W, Khalili K. Negative feedback regulation of HIV-1 by gene editing strategy. Sci Rep. 2016c; 6:31527. [PubMed: 27528385]

Karvelis T, Gasiunas G, Young J, Bigelyte G, Silanskas A, Cigan M, Siksnys V. Rapid characterization of CRISPR-Cas9 protospacer adjacent motif sequence elements. Genome Biol. 2015; 16:253. [PubMed: 26585795]

Kennedy EM, Cullen BR. Gene Editing: A New Tool for Viral Disease. Annu Rev Med. 2017; 68:401411. [PubMed: 27576009]

Khalili K, Kaminski R, Gordon J, Cosentino L, Hu W. Genome editing strategies: potential tools for eradicating HIV-1/AIDS. J Neurovirol. 2015; 21:310-321. [PubMed: 25716921]

Khalili K, White MK, Jacobson JM. Novel AIDS therapies based on gene editing. Cell Mol Life Sci. 2017 (In Press).

Kim E, Koo T, Park SW, Kim D, Kim K, Cho HY, Song DW, Lee KJ, Jung MH, Kim S, Kim JH, Kim JH, Kim JS. In vivo genome editing with a small Cas9 orthologue derived from Campylobacter jejuni. Nat Commun. 2017 (In Press).

Kleinstiver BP, Prew MS, Tsai SQ, Topkar VV, Nguyen NT, Zheng Z, Gonzales AP, Li Z, Peterson RT, Yeh JR, Aryee MJ, Joung JK. Engineered CRISPR-Cas9 nucleases with altered PAM specificities. Nature. 2015; 523:481-485. [PubMed: 26098369]

Kleinstiver BP, Pattanayak V, Prew MS, Tsai SQ, Nguyen NT, Zheng Z, Joung JK. High-fidelity CRISPR-Cas9 nucleases with no detectable genome-wide off-target effects. Nature. 2016; 529:490-495. [PubMed: 26735016]

Komor AC, Kim YB, Packer MS, Zuris JA, Liu DR. Programmable editing of a target base in genomic DNA without double-stranded DNA cleavage. Nature. 2016; 533:420-424. [PubMed: 27096365]

Komor AC, Badran AH, Liu DR. CRISPR-Based technologies for the manipulation of eukaryotic genomes. Cell. 2017; 168:20-36. [PubMed: 27866654]

Kotterman MA, Chalberg TW, Schaffer DV. Viral vectors for gene therapy: translational and clinical outlook. Annu Rev Biomed Eng. 2015; 17:63-89. [PubMed: 26643018]

Liao HK, Gu Y, Diaz A, Marlett J, Takahashi Y, Li M, Suzuki K, Xu R, Hishida T, Chang CJ, Esteban CR, Young J, Izpisua Belmonte JC. Use of the CRISPR/Cas9 system as an intracellular defense against HIV-1 infection in human cells. Nat Commun. 2015; 6:6413. [PubMed: 25752527]

Li Z, Bi Y, Xiao H, Sun L, Ren Y, Li Y, Chen C, Cun W. CRISPR-Cas9 system-driven site-specific selection pressure on herpes simplex virus genomes. Virus Res. 2017 pii: S0168-1702(16)30694-3.

Liang X, Potter J, Kumar S, Zou Y, Quintanilla R, Sridharan M, Carte J, Chen W, Roark N, Ranganathan S, Ravinder N, Chesnut JD. Rapid and highly efficient mammalian cell engineering via Cas9 protein transfection. J Biotechnol. 2015; 208:44-53. [PubMed: 26003884]

Liu J, Shui SL. Delivery methods for site-specific nucleases: Achieving the full potential of therapeutic gene editing. J Control Release. 2016; 244(Pt A):83-97. [PubMed: 27865852]

Lotti SN, Polkoff KM, Rubessa M, Wheeler MB. Modification of the genome of domestic animals. Anim Biotechnol. 2017; 19:1-13.

Low BE, Kutny PM, Wiles MV. Simple, efficient CRISPR-Cas9-mediated gene editing in mice: strategies and methods. Methods Mol Biol. 2016; 1438:19-53. [PubMed: 27150082]

McGeoch DJ, Rixon FJ, Davison AJ. Topics in herpesvirus genomics and evolution. Topics in herpesvirus genomics and evolution. Virus Res. 2006; 117:90-104. [PubMed: 16490275]

Mohanraju P, Makarova KS, Zetsche B, Zhang F, Koonin EV, van der Oost J. Diverse evolutionary roots and mechanistic variations of the CRISPR-Cas systems. Science. 2016; 353:aad5147. [PubMed: 27493190] 
Moyo B, Bloom K, Scott T, Ely A, Arbuthnot P. Advances with using CRISPR/Cas-mediated gene editing to treat infections with hepatitis B virus and hepatitis C virus. Virus Res. 20172017 Jan 10. pii:S0168-1702(16)30733-X.

Nakade S, Yamamoto T, Sakuma T. Cas9, Cpf1 and C2c1/2/3-What's next? Bioengineered. 2017 (In Press).

Nishida K, Arazoe T, Yachie N, Banno S, Kakimoto M, Tabata M, Mochizuki M, Miyabe A, Araki M, Hara KY, Shimatani Z, Kondo A. Targeted nucleotide editing using hybrid prokaryotic and vertebrate adaptive immune systems. Science. 2016; 353 pii:aaf8729.

O'Connell MR, Oakes BL, Sternberg SH, East-Seletsky A, Kaplan M, Doudna JA. Programmable RNA recognition and cleavage by CRISPR/Cas9. Nature. 2014; 516:263-266. [PubMed: 25274302]

Price AA, Sampson TR, Ratner HK, Grakoui A, Weiss DS. Cas9-mediated targeting of viral RNA in eukaryotic cells. Proc Natl Acad Sci USA. 2015; 112:6164-619. [PubMed: 25918406]

Ran FA. Adaptation of CRISPR nucleases for eukaryotic applications. Anal Biochem. 20162016. pii:S0003-2697(16)30354-2.

Ran FA, Cong L, Yan WX, Scott DA, Gootenberg JS, Kriz AJ, Zetsche B, Shalem O, Wu X, Makarova KS, Koonin EV, Sharp PA, Zhang F. In vivo genome editing using Staphylococcus aureus Cas9. Nature. 2015; 520:186-191. [PubMed: 25830891]

Rauch BJ, Silvis MR, Hultquist JF, Waters CS, McGregor MJ, Krogan NJ, Bondy-Denomy J. Inhibition of CRISPR-Cas9 with bacteriophage proteins. Cell. 2017; 168:150-158. [PubMed: 28041849]

Roehm PC, Shekarabi M, Wollebo HS, Bellizzi A, He L, Salkind J, Khalili K. Inhibition of HSV-1 replication by gene editing strategy. Sci Rep. 2016; 6:23146. [PubMed: 27064617]

Seth K, Harish. Current status of potential applications of repurposed Cas9 for structural and functional genomics of plants. Biochem Biophys Res Commun. 2016; 480:499-507. [PubMed: 27955725]

Shalem O, Sanjana NE, Zhang F. High-throughput functional genomics using CRISPR-Cas9. Nat Rev Genet. 2015; 16:299-311. [PubMed: 25854182]

Shmakov S, Abudayyeh OO, Makarova KS, Wolf YI, Gootenberg JS, Semenova E, Minakhin L, Joung J, Konermann S, Severinov K, Zhang F, Koonin EV. Discovery and functional characterization of diverse class 2 CRISPR-Cas systems. Mol Cell. 2015; 60:385-397. [PubMed: 26593719]

Senís E, Fatouros C, Große S, Wiedtke E, Niopek D, Mueller AK, Börner K, Grimm D. CRISPR/ Cas9-mediated genome engineering: an adeno-associated viral (AAV) vector toolbox. Biotechnol J. 2014; 9:1402-1412. [PubMed: 25186301]

Singh V, Braddick D, Dhar PK. Exploring the potential of genome editing CRISPR-Cas9 technology. Gene. 2017; 599:1-18. [PubMed: 27836667]

Slaymaker IM, Gao L, Zetsche B, Scott DA, Yan WX, Zhang F. Rationally engineered Cas9 nucleases with improved specificity. Science. 2016; 351:84-88. [PubMed: 26628643]

Tsai SQ, Joung JK. Defining and improving the genome-wide specificities of CRISPR-Cas9 nucleases. Nat Rev Genet. 2016; 17:300-312. [PubMed: 27087594]

Tschaharganeh DF, Lowe SW, Garippa RJ, Livshits G. Using CRISPR/Cas to study gene function and model disease in vivo. FEBS J. 2016; 283:3194-3203. [PubMed: 27149548]

Tycko J, Myer VE, Hsu PD. Methods for optimizing CRISPR-Cas9 genome editing specificity. Mol Cell. 2016; 63:355-370. [PubMed: 27494557]

van Diemen FR, Kruse EM, Hooykaas MJ, Bruggeling CE, Schürch AC, van Ham PM, Imhof SM, Nijhuis M, Wiertz EJ, Lebbink RJ. PLoS Pathog. 2016; 12:e1005701. [PubMed: 27362483]

Wang G, Zhao N, Berkhout B, Das AT. CRISPR-Cas9 can inhibit HIV-1 replication but NHEJ repair facilitates virus escape. Mol Ther. 2016a; 24:522-526. [PubMed: 26796669]

Wang L, Li F, Dang L, Liang C, Wang C, He B, Liu J, Li D, Wu X, Xu X, Lu A, Zhang G. In Vivo delivery systems for therapeutic genome editing. Int J Mol Sci. 2016b; 17 pii:E626.

Wang M, Zuris JA, Meng F, Rees H, Sun S, Deng P, Han Y, Gao X, Pouli D, Wu Q, Georgakoudi I, Liu $\mathrm{DR}, \mathrm{Xu} \mathrm{Q}$. Efficient delivery of genome-editing proteins using bioreducible lipid nanoparticles. Proc Natl Acad Sci USA. 2016c; 113:2868-2873. [PubMed: 26929348] 
Wang X, Huang X, Fang X, Zhang Y, Wang W. CRISPR-Cas9 system as a versatile tool for genome engineering in human cells. Mol Ther Nucleic Acids. 2016d; 5:e388. [PubMed: 27845770]

Wang Z, Pan Q, Gendron P, Zhu W, Guo F, Cen S, Wainberg MA, Liang C. CRISPR/Cas9-derived mutations both inhibit HIV-1 replication and accelerate viral escape. Cell Rep. 2016e; 15:481-489. [PubMed: 27068471]

White MK, Khalili K. CRISPR/Cas9 and cancer targets: future possibilities and present challenges. Oncotarget. 2016; 7:12305-12317. [PubMed: 26840090]

White MK, Khalili K. Strategy optimization of gene editing approaches against viral infections to prevent viral escape. PLoS Pathog. 2016; 12:e1005953. [PubMed: 27930735]

White MK, Hu W, Khalili K. The CRISPR/Cas9 genome editing methodology as a weapon against human viruses. Discov Med. 2015; 19:255-262. [PubMed: 25977188]

White MK, Kaminski R, Wollebo H, Hu W, Malcolm T, Khalili K. Gene editing for treatment of neurological infections. Neurotherapeutics. 2016a; 13:547-554. [PubMed: 27150390]

Wollebo HS, Bellizzi A, Kaminski R, Hu W, White MK, Khalili K. CRISPR/Cas9 system as an agent for eliminating Polyomavirus JC infection. PLoS One. 2015a; 10:e0136046. [PubMed: 26360417]

Wollebo HS, White MK, Gordon J, Berger JR, Khalili K. Persistence and pathogenesis of the neurotropic polyomavirus JC. Ann Neurol. 2015b; 77:560-570. [PubMed: 25623836]

Wright DA, Li T, Yang B, Spalding MH. TALEN-mediated genome editing: prospects and perspectives. Biochem J. 2014; 462:15-24. [PubMed: 25057889]

Wright AV, Nuñez JK, Doudna JA. Biology and applications of CRISPR systems: harnessing nature's toolbox for genome engineering. Cell. 2016; 164:29-44. [PubMed: 26771484]

Xu X, Tay Y, Sim B, Yoon SI, Huang Y, Ooi J, Utami KH, Ziaei A, Ng B, Radulescu C, Low D, Ng AY, Loh M, Venkatesh B, Ginhoux F, Augustine GJ, Pouladi MA. Reversal of phenotypic abnormalities by CRISPR/Cas9-mediated gene correction in Huntington disease patient-derived induced pluripotent stem cells. Stem Cell Reports. 2017; 8:619-633. [PubMed: 28238795]

Ye L, Wang J, Beyer AI, Teque F, Cradick TJ, Qi Z, Chang JC, Bao G, Muench MO, Yu J, Levy JA, Kan YW. Seamless modification of wild-type induced pluripotent stem cells to the natural CCR5 32 mutation confers resistance to HIV infection. Proc Natl Acad Sci USA. 2014; 111:9591-9596. [PubMed: 24927590]

Yee JK. Off-target effects of engineered nucleases. FEBS J. 2016; 283:3239-3248. [PubMed: 27208701]

Yin C, Zhang T, Li F, Yang F, Putatunda R, Young WB, Khalili K, Hu W, Zhang Y. Functional screening of guide RNAs targeting the regulatory and structural HIV-1 viral genome for a cure of AIDS. AIDS. 2016; 30:1163-1174. [PubMed: 26990633]

Yin C, Zhang T, Qu X, Zhang Y, Putatunda R, Xiao X, Li F, Xiao W, Zhao H, Dai S, Qin X, Mo X, Young WB, Khalili K, Hu W. In vivo excision of HIV-1 provirus by saCas9 and multiplex sgRNAs in animal models. Molecular Therapy. 2017 (in press).

Yu W, Mookherjee S, Chaitankar V, Hiriyanna S, Kim JW, Brooks M, Ataeijannati Y, Sun X, Dong L, Li T, Swaroop A, Wu Z. Nrl knockdown by AAV-delivered CRISPR/Cas9 prevents retinal degeneration in mice. Nat Commun. 2017; 8:14716. [PubMed: 28291770]

Yuen KS, Chan CP, Kok KH, Jin DY. Mutagenesis and genome engineering of Epstein-Barr Virus in cultured human cells by CRISPR/Cas9. Methods Mol Biol. 2017; 1498:23-31. [PubMed: 27709566]

Zetsche B, Gootenberg JS, Abudayyeh OO, Slaymaker IM, Makarova KS, Essletzbichler P, Volz SE, Joung J, van der Oost J, Regev A, Koonin EV, Zhang F. Cpf1 is a single RNA-guided endonuclease of a class 2 CRISPR-Cas system. Cell. 2015; 163:759-771. [PubMed: 26422227]

Zhang Y, Yin C, Zhang T, Li F, Yang W, Kaminski R, Fagan PR, Putatunda R, Young WB, Khalili K, $\mathrm{Hu}$ W. CRISPR/gRNA-directed synergistic activation mediator (SAM) induces specific, persistent and robust reactivation of the HIV-1 latent reservoirs. Sci Rep. 2015a; 5:16277. [PubMed: 26538064]

Zhang XH, Tee LY, Wang XG, Huang QS, Yang SH. Off-target effects in CRISPR/Cas9-mediated genome engineering. Mol Ther Nucleic Acids. 2015b; 4:e264. [PubMed: 26575098] 
Zischewski J, Fischer R, Bortesi L. Detection of on-target and off-target mutations generated by CRISPR/Cas9 and other sequence-specific nucleases. Biotechnol Adv. 2017; 35:95-104.

[PubMed: 28011075] 


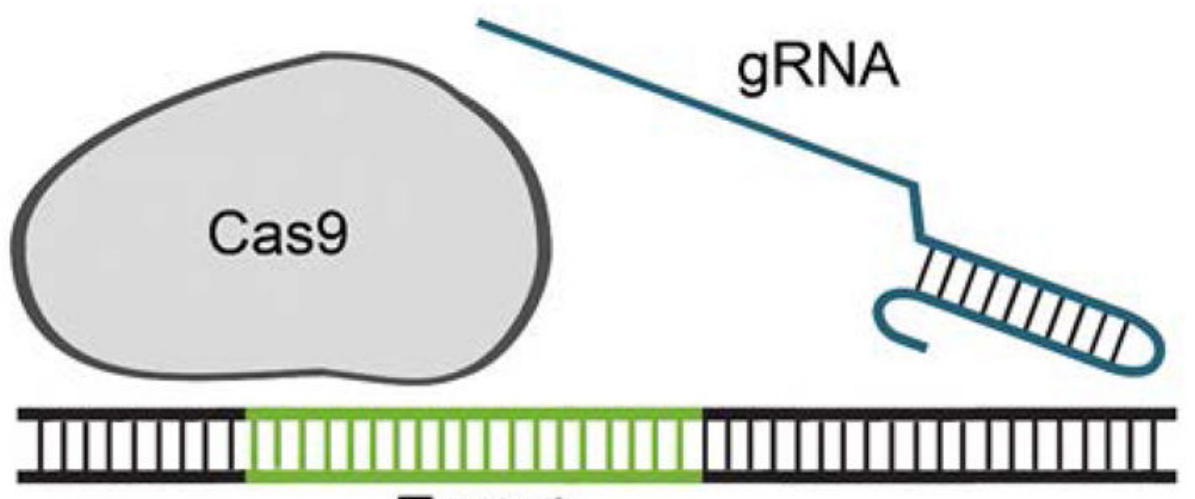

Target

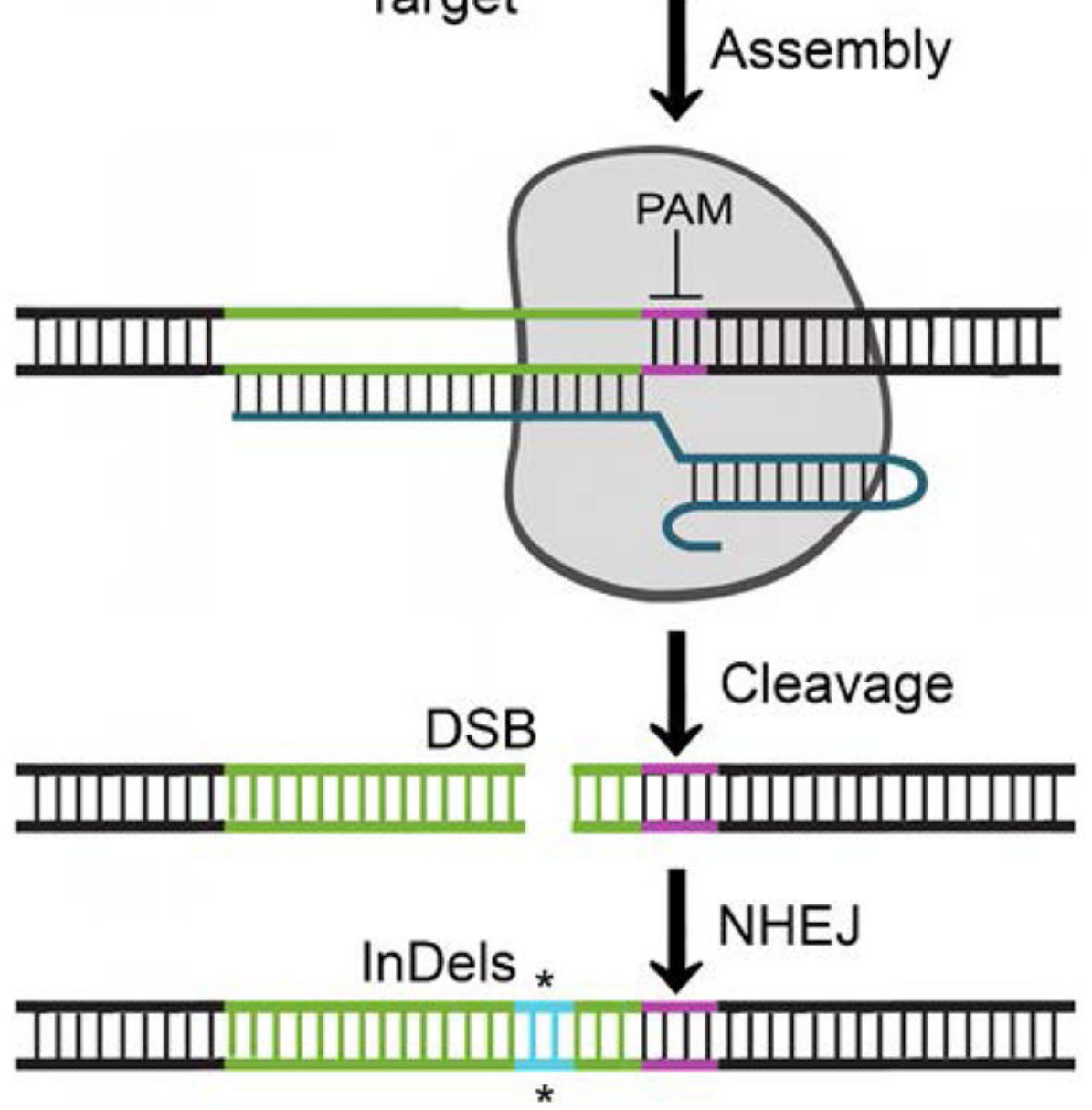

Figure 1. Schematic of CRISPR/Cas9-mediated gene inactivation

The schematic shows the action of Cas9 endonuclease on its target DNA (shown in green) and target-specific gRNA (shown in dark blue). In the first step, Cas9 binds to the target DNA guided by its complementarity to the DNA target and the presence of the PAM sequence. PAM is the protospacer adjacent motif and is shown in purple. In the second step, the target DNA undergoes endonucleolytic cleavage catalyzed by Cas9 to give a doublestrand break (DSB). In the third step, the DSB is repaired by nonhomologous end-joining (NHEJ), which is an error-prone form of DNA repair that may allow the introduction of 
Insertion/Deletion mutations (InDels) that can impair the function of the gene (shown in bright blue and marked *). 


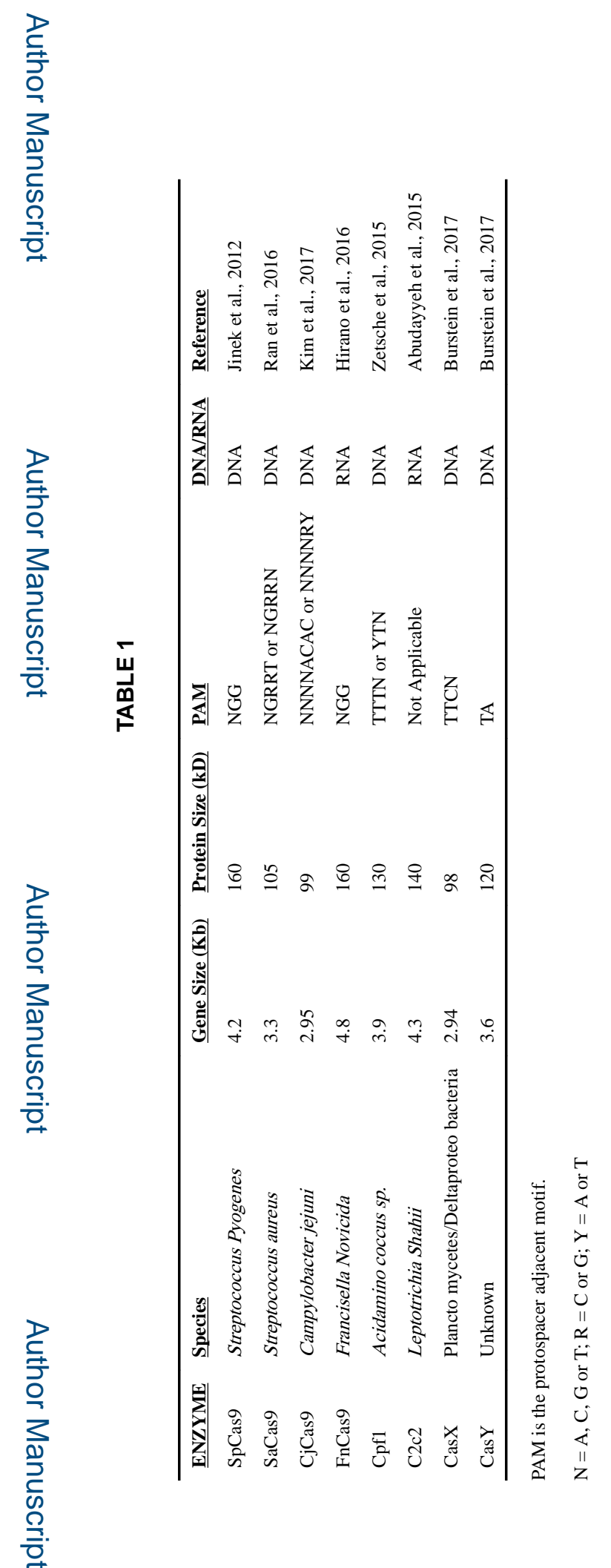

J Cell Biochem. Author manuscript; available in PMC 2018 November 01. 